\title{
Time to Recurrence of any Opportunistic Infection after Treatment of it among People Living with HIV Infection in Debre Markos, Northwest Ethiopia: Retrospective Cohort Study
}

Habtamu Mellie ${ }^{1}$, Getnet Mitike ${ }^{2}$ and Amanuel Alemu Abajobir ${ }^{1 *}$

${ }^{1}$ Debre Markos University, Department of Public Health, Debre Markos, Ethiopia

${ }^{2}$ Addis Ababa Universities, School of Public Health, Addis Ababa, Ethiopia

\begin{abstract}
Introduction: Antiretroviral therapy improves time to death of people living with HIV; however, little is known about its effect on improving time of recurrence of opportunistic infections after treatment of any preceding infection. Hence, this study was aimed at assessing time to recurrence of any opportunistic infection after treatment of any preceding opportunistic infection, its magnitude and associated factors.

Methods: Retrospective cohort study was used and the required sample size was 536 . Study participants were selected randomly from the list of adult people living with HIV attending the public health facilities for ART. Univariate analysis was used to describe patients' baseline and follow up characteristics. Kaplan-meier survival and log rank test were used to estimate survival and compare survival curves respectively. Cox proportional-hazard regression model was used to calculate hazard rate and to determine independent predictors of time to recurrence.
\end{abstract}

Results: During a median of 43 person weeks follow up, opportunistic infections recurred in $75.7 \%$ the participants and the proportion was not significantly different $(p=0.614)$ in pre-ART and ART people living with HIV. The incidence rate of any opportunistic infection recurrence was 1.31 per 100 person weeks which differs by ART exposure status which was 1.57 and 1.13 per 100 person weeks in pre-ART and ART PLHIV respectively. The median time of survival was 57 weeks; however, it was different in pre-ART and ART PLHIV that was 52 and 64 weeks respectively. Marital status, occupational status, follow up CD4 count, base line hemoglobin value, ART exposure, base line ART adherence, base line and follow up prophylaxis exposure and adherence were predictors of survival.

Conclusion: The incidence rate of any opportunistic infection recurrence was 1.31 per 100 person weeks. Attention should be given for widowed, ART/prophylaxis non-adhering patients while continuing HIV care for all people living with the virus.

Keywords: HIV; Recurrence; Opportunistic infection

\section{Introduction}

According to the latest United Nation's Agency for International Development (UNAID) estimate, about 34 million people were living with human immunodeficiency virus (HIV) in 2011 [1,2]. Globally, the annual number of people dying from acquired immunodeficiency syndrome (AIDS)-related causes was 1.7 million in 2011 [3].

According to 2011 Ethiopian demographic health survey, the overall national adult (age 15-49 years) HIV prevalence was 1.5\% [4].

Introduction of combination antiretroviral therapy in 1996 has led to dramatic reductions in morbidity and mortality from HIV/AIDS $[1,2]$. In Ethiopia, the fee based and universal free access antiretroviral (ARV) treatment was started in 2002/3 and 2004/5 respectively [5,6].

HIV infection leads to AIDS and major causes of morbidity and mortality of such patients are opportunistic infections (OIs) caused by viral, bacterial, fungal and parasitic pathogens [7]. OIs can occur in about $40 \%$ of people living with HIV (PLHIV) with a CD4 count less than $250 / \mathrm{mm}^{3}$ [8].

In Northwest Ethiopia, a study at Felegehiwot hospital in pre-ART HIV/AIDS patients showed that $88.9 \%$ of them have common OIs (chronic diarrhea (38.6\%), tuberculosis (TB) (34.5\%), herpes zoster (30.7\%), oro-pharyngeal candidiasis (14\%), pneumonia $(3.5 \%)$ and other sexually transmitted infections (12.5\%)) [9].

The other problems among the HIV sero-positive individuals were dual and triple OIs and other co-infections; according to studies, the most frequent dual infections were chronic diarrhea and oral candidiasis (28.92\%), oral candidiasis and TB (25.49\%), chronic diarrhea and TB (21.08\%), HSV-2 and oral candidiasis (19.11\%), HSV2 and cytomegaly virus (14.21\%), Hepatitis-B virus and HSV-2 (3.92\%); the commonest triple infections were oral candidiasis, TB and chronic diarrhea (14.21\%); HSV-2, CMV and oral candidiasis (10.78\%); HSV-2, CMV and chronic diarrhea (8.34\%); and HSV-2, CMV and TB (6.86\%) [10].

Different studies showed that ART improves time to death among PLHIV [11-13] but its effect on improving time of recurrence of any OI after treatment of any preceding OI, its magnitude and associated factors are not studied in Ethiopia. Hence, this study was aimed at assessing time to recurrence of any OI after treatment of any preceding OI, its magnitude and associated factors.

*Corresponding author: Amanuel Alemu Abajobir, Debre Markos University, Department of Public Health, Debre Markos, Ethiopia, E-mail: habtamumellie@yahoo.com; amanuel_alemu@yahoo.com

Received June 17, 2013; Accepted July 25, 2013; Published July 31, 2013

Citation: Mellie H, Mitike G, Abajobir AA (2013) Time to Recurrence of any Opportunistic Infection after Treatment of it among People Living with HIV Infection in Debre Markos, Northwest Ethiopia: Retrospective Cohort Study. J AIDS Clin Res 4 226. doi:10.4172/2155-6113.1000226

Copyright: ( 2013 Mellie H, et al. This is an open-access article distributed unde the terms of the Creative Commons Attribution License, which permits unrestricted use, distribution, and reproduction in any medium, provided the original author and source are credited. 


\section{Methods and Patients}

\section{Study area, period and design}

Debre Markos town has one referral hospital and one public health center, which provide ART service. The study was conducted in Debre Markos hospital and health center among 6261 adult PLHIV who were on chronic HIV care or ART follow up between $25^{\text {th }}$ March 2007 and $24^{\text {th }}$ March 2013. Institution-based retrospective cohort study was conducted from March $25^{\text {th }} 2007$ to March $24^{\text {th }}$ using quantitative research method.

\section{Source and study population}

All 18 years old and above PLHIV infections having chronic HIV care or on ART follow up in Debre Markos town health institutions providing ART service were the source populations. All adult PLHIV infections that had developed any OI and on standard treatment in chronic HIV care or on ART follow up found in ART service providing health institutions in Debre Markos town were included in the study

\section{Sampling and sample size determination}

The sample size was calculated based on the assumption of $95 \%$ confidence interval and $80 \%$ power with ratio of one to one for ART and pre-ART PLHIV using the proportions of the commonest OI (TB) which were $6 \%$ and $14 \%$ for ART and pre-ART PLHIV respectively [11]. Epi Info version 3.5 was used to calculate the required sample size [14].

$$
\begin{aligned}
& \mathrm{n}=\frac{\left[\left[Z_{\alpha} \sqrt{(1+1 / \mathrm{m}) \overline{\mathrm{p}}(1-\overline{\mathrm{p}})}+\mathrm{Z}_{\beta} \sqrt{\mathrm{p}_{\mathrm{o}}\left(1-\mathrm{p}_{\mathrm{o}}\right) / \mathrm{m}+\mathrm{p}_{1}\left(1-\mathrm{p}_{1}\right)}\right]^{2}\right.}{\left(\mathrm{p}_{\mathrm{o}}-\mathrm{p}_{1}\right)^{2}} \\
& \overline{\mathrm{p}}=\frac{\mathrm{p} 1+\mathrm{mp}_{\mathrm{o}}}{\mathrm{m}+1}
\end{aligned}
$$

where, $\mathrm{n}$ : minimum sample size required for each group

m: ratio of pre-ART and ART PLHIV

$\mathrm{p}_{\mathrm{o}}$ : probability of event in pre-ART

$\mathrm{p}_{1}$ : probability of event in ART

a: level of significance

$\mathrm{Z}_{\alpha / 2:} 1.96$ at $95 \%$ confidence interval

Power: $1-\beta=80 \%, Z_{\beta}=1.282$

Accordingly, the calculated sample size was 488 (244 for ART and pre-ART); after adding $10 \%$ contingency the sample was 536 . Eligible study participants were identified by data collectors from the list of PLHIV who were on HIV care or on ART follow up from ART clinics of Debre Markos hospital and health center. Eligible PLHIV who were on pre-ART and ART history were given separate codes and then selected by simple random sampling using random number table. Moreover, in order to reduce falsely survival increment, study participants were asked by data collectors about treatment history for OIs.

\section{Data collection instrument and procedure}

Data abstraction format was developed from federal ministry of health HIV care/ART follow up form which is used in the ART clinic and also the patients' card; the format includes the following check list:

$>$ socio-demographic characteristics (sex, age, residence, marital status, occupational status, educational status);

> ART and chemoprophylaxis information (ARV drug treatment, drug adherence, line of treatment regimen, prophylaxis treatment and its adherence);

$>$ laboratory information (CD4 count, hemoglobin value);

$>$ Clinical information (WHO clinical staging, weight, height, OIs (name and count treated at one time), other chronic diseases like diabetes mellitus, hypertension, cardiac disease); and

Functional status of patients (working, ambulatory, bedridden).

The data were collected by reviewing HIV care/ART follow up form, laboratory requests and patients' card. If PLHIV develop any OI before completing treatment of any preceding OI while the patient is on study, the time of treatment, which is nearest to, end of the study was used as the starting time for study. If laboratory results (CD4 count, $\mathrm{Hgb}$ ) were not found during OI treatment, the most recent laboratory results before treatment of OI were used as base line predictors. Length of follow up for each study participant was varied because of different enrollment time.

Reoccurrence of any OI was confirmed by reviewing HIV care/ ART follow-up form or patient card or by asking study participant during data collection period about treatment history for OIs out of the follow up health institution. The health status was assured by asking the study participants when she or he came to ART clinic for follow up, asking using registered address on follow up form like phone number, kebele, house number, or adherence supporter groups were used to get their homes and used to trace study participants.

Individuals who change from unexposed to exposed, drop-out/loss to follow-up/transferred out/dead by any disease other than OI where cause of death not confirmed during study period or not develop any OI at the end of the study period were censored. The outcome of each subject was dichotomized into censored or recurrence of any OI.

Three data collectors and one supervisor who had direct experience and working on ART clinic were recruited for data collection and supervision respectively. Data collectors and supervisor were trained on objectives of the study, selection of exposed and unexposed, confidentiality of information, the contents of the questionnaire and data quality management by the principal investigators.

\section{Data quality management, processing and analysis}

To maintain data quality, incompletely recorded follow up formats were excluded from abstraction to check the reliability and consistency of data and accordingly correction was made. Data cleaning and editing were made before analysis.

Each abstraction format was given a code and was entered in to Epi Info version 3.35 statistical software and exported to SPSS 16 and STATA 11 statistical packages for analysis of statistical inferences.

The patient cohort characteristics including age, CD4 count, Hgb value, time to reoccurrence of any OIs and body mass index (BMI) were described in terms of mean or median value and other characteristics like sex, residence, marital status, occupational status, educational status, WHO clinical staging, ART adherence, line of ART regimen, prophylaxis treatment and its adherence, functional status, final outcome of study (censored or reoccurrence of any OIs) were described in terms percentages, tables and graphs.

Proportion and incidence of any OI were calculated at the end of the study. Incidence rate was calculated by dividing total events to personweeks. The actuarial life table and Kaplan Meier survival was used to 
Citation: Mellie H, Mitike G, Abajobir AA (2013) Time to Recurrence of any Opportunistic Infection after Treatment of it among People Living with HIV Infection in Debre Markos, Northwest Ethiopia: Retrospective Cohort Study. J AIDS Clin Res 4: 226. doi:10.4172/2155-6113.1000226

Page 3 of 10

estimate survival time and log rank test was used for categorical variables to compare survival curves across each strata. Before running the Cox regression model assumption of proportional-hazard was checked by Schoenfeld residual with $p$-value $\geq 0.1(\alpha=10 \%)$ and the assumption was not violated. Multi-colinearity was checked using Pearson correlation, tolerance or variance inflation factor and we found that base line ART regimen was highly correlated with follow up ART regimen ( $\mathrm{r}=0.809$, $\mathrm{p}<0.0001$ ) so that further analysis in the final model were not done for base line ART regimen. Similarly, follow up Hgb value were excluded in final model because of its correlation with base line $\mathrm{Hgb}$ value $(\mathrm{r}=0.598$, $\mathrm{p}<0.0001)$ and it was done in only $10.8 \%$ of study participants. Exclusion of redundant variables was done to stabilize final model and to improve precision of estimation. Cox proportional-hazard model was used to calculate the univariate and multivariable adjusted hazard rate and to determine independent predictors of survival. In Multivariable cox proportional hazard model, only those variables which were associated with time to recurrence of any OI with $p$-value $\leq 0.2$ in univariate analysis and not collinear were entered to the final model.

\section{Study variables}

\section{Dependent variable}

$>$ Time to recurrence of any OIs after its treatment

\section{Independent variables}

$>$ socio-demographic (age, sex, religion, residence, occupation, educational status, marital status)

$>$ clinical, laboratory, ART and chemoprophylaxis information and functional status (working, ambulatory, bedridden)

\section{Definitions}

Pre-ART PLHIV: The person whose HIV positive status confirmed but not eligible for ART;

ART PLHIV: The person whose HIV positive status confirmed and started ART;
Survival: The person without any OI recurrence;

Censored: Non-recurrence of any OI in study participant during follow up on study but not sure for future recurrence;

Reoccurrence: The happening or diagnosis of any OI by health personnel working in ART health center. Confidentiality and anonymity was maintained. Informed consent was clinic after completing the preceding treatment of any OI;

Drop out: If a PLHIV on HIV care lost to follow up for more than three months as recorded by ART clinic personnel;

Lost to follow up: If PLHIV on HIV care not seen for equal to or more than one month as recorded by ART clinic personnel;

Transferred out: If PLHIV on HIV care in one health institution shift to other health institution;

Good Adherence: if adherent $\geq 95 \%$; that is the percentage of missed dose is $<2$ doses of 30 doses or $<3$ dose of 60 dose) as documented by ART health personnel;

Fair Adherence: if adherent 85-94\%; that is the percentage of missed dose is 3-5 doses of 30 doses or 3-9 dose of 60 dose) as documented by ART health personnel;

Poor Adherence: if adherent $<85 \%$; that is the percentage of missed dose is $\geq 3$ doses of 30 doses or $>9$ dose of 60 dose) as documented by ART health personnel.

\section{Ethical consideration}

Ethical approval and clearance were obtained from research and ethical committee of School of Public Health of Addis Ababa University. Permission was also obtained from the concerned bodies of the hospital and obtained from study participants to get permission to extract data from their medical records. To get study participants who were not coming to the ART clinic for follow up during data collection period, in addition of using already registered address like phone number, kebele, house number on follow up form and ART adherence supporter groups

\begin{tabular}{|c|c|c|c|c|c|c|c|}
\hline \multirow{2}{*}{ Recurred OI } & \multicolumn{2}{|c|}{ Pre-ART (268) } & \multicolumn{2}{|c|}{ ART (268) } & \multirow{2}{*}{$X^{2} p$-value } & \multicolumn{2}{|c|}{ PLHIV (536) } \\
\hline & Frequency (\%) & IR & Frequency (\%) & IR & & Frequency (\%) & IR \\
\hline Recurrent URTIs & $35(13.1)$ & 0.27 & $38(14.2)$ & 0.21 & 0.91 & $73(13.6)$ & 0.24 \\
\hline Chronic diarrhea & $30(11.2)$ & 0.23 & $22(8.2)$ & 0.12 & 0.25 & $52(9.7)$ & 0.17 \\
\hline Pneumonia & $21(7.8)$ & 0.16 & $25(9.3)$ & 0.14 & 0.73 & $46(8.5)$ & 0.15 \\
\hline Oral candidacies & $22(8.2)$ & 0.17 & $21(7.8)$ & 0.12 & 0.91 & $43(8.0)$ & 0.14 \\
\hline Herpes zoster & $18(6.7)$ & 0.14 & $20(7.5)$ & 0.11 & 0.81 & $38(7.1)$ & 0.12 \\
\hline EPTB & $15(5.6)$ & 0.12 & $16(6.0)$ & 0.09 & 0.92 & $31(5.8)$ & 0.10 \\
\hline Minor MC manifestations & $13(4.9)$ & 0.10 & $15(5.6)$ & 0.08 & 0.92 & $28(5.2)$ & 0.09 \\
\hline PTB & $10(3.7)$ & 0.08 & $13(4.9)$ & 0.07 & 0.72 & $23(4.2)$ & 0.07 \\
\hline PCP & $12(4.5)$ & 0.09 & $9(3.4)$ & 0.05 & 0.61 & $21(3.9)$ & 0.07 \\
\hline Genital/oral ulcer & $8(3.0)$ & 0.06 & $6(2.2)$ & 0.03 & 0.75 & $14(2.6)$ & 0.05 \\
\hline Toxoplasmosis & $6(2.2)$ & 0.05 & $5(1.9)$ & 0.03 & 0.95 & $11(2.1)$ & 0.04 \\
\hline Encephalopathy & $4(1.5)$ & 0.03 & $7(2.6)$ & 0.04 & 0.57 & $11(2.1)$ & 0.04 \\
\hline Wasting syndrome & $8(3.0)$ & 0.06 & $3(1.1)$ & 0.02 & 0.20 & $11(2.1)$ & 0.04 \\
\hline Cryptococal meningitis & $3(1.1)$ & 0.02 & $8(3.0)$ & 0.04 & 0.24 & $11(2.1)$ & 0.04 \\
\hline Persistent generalized LAP & $6(2.2)$ & 0.05 & $3(1.1)$ & 0.02 & $0.33^{*}$ & $9(1.7)$ & 0.03 \\
\hline Others & $7(2.6)$ & 0.06 & $9(3.4)$ & 0.05 & 0.78 & $16(3.0)$ & 0.05 \\
\hline Total $\#$ & $200(74.6)$ & 1.57 & $206(76.9)$ & 1.13 & 0.61 & $406(75.7)$ & 1.31 \\
\hline
\end{tabular}

IR: incidence rate per 100 person weeks; MC: muco-cutaneous; LAP: lymphadenopathy; URTIs: upper respiratory tract infections; PTB: pulmonary tuberculosis; EPTB: extra pulmonary tuberculosis; PCP: pneumocystis Carinii pneumonia; Row total is not sum of columns values due to occurrence of more than one OI in some study participants.

* P-value is Fisher's Exact test because of $50 \%$ of the expected cell values are less than 5 .

Table 1: Proportion and incidence of recurred Ols in pre-ART and ART PLHIV in Debre Markos Hospital and Health Center, Northwest Ethiopia between $25^{\text {th }}$ March 2007 and $24^{\text {th }}$ March, 2013. 


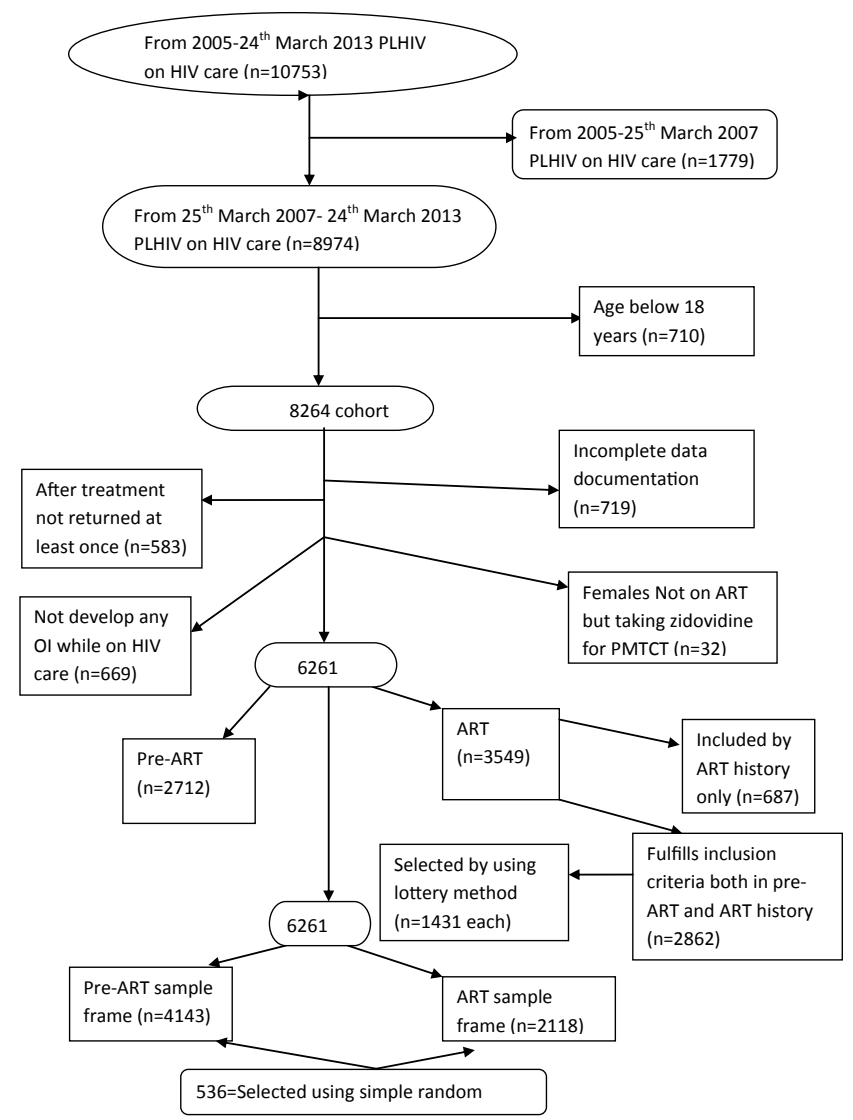

Figure 1: Profile of PLHIV enrolled for cohort between $25^{\text {th }}$ March 2007 and $24^{\text {th }}$ March 2013 in Debre Markos Hospital and Health Center, Northwest Ethiopia, 2013

were used to trace them. For study participants who were severely ill, with coma or having hearing impairment or died before data collection period while included in the study period, the nearest relatives (family) or legal representatives were asked to give permission to extract on medical records.

\section{Results}

In this historical cohort study, between $25^{\text {th }}$ March 2007 and $24^{\text {th }}$ March 2013, 8,974 PLHIV aged 18 years and above were evaluated (Figure 1). Five hundred thirty six PLHIV who were on pre-ART and ART with 1:1 ratio each consisting 268 were followed for a total of 30971 person weeks and median of 43 person weeks. The minimum and maximum person week follow up was 2 and 258 respectively. The study assessed time to recurrence of any OI in pre-ART and ART PLHIV, its magnitude and baseline and follow up predictors of time to recurrence in PLHIV.

\section{Socio demographic characteristics}

In this study, $354(66 \%)$ females and $182(34 \%)$ males were participated. Their median age was 32 year $(\mathrm{IQR}=26-40)$ in which 493 (92\%) were below 50 year of age. Most of the study participants were urban by residence $376(70.1 \%)$, orthodox by religion 495 (92.4\%), not educated 236 (44\%), married 232 (43.3\%) and employed 107 (20\%) (Table 3).

\section{Clinical, laboratory and treatment information}

At base line, three quarter of the study participants (400, 74.6\%) had one episode of OI and most $(214,39.9 \%)$ of them had WHO Clinical stage III. Almost all of the study participants $(394,97.1 \%)$ had no any chronic diseases like hypertension, diabetes mellitus and others.

With regard to functional status, more than three quarters of the participants were working both at base line $(418,78 \%)$ and at follow up $(471,87.1 \%)$.

The base line and end line mean (SD) values for BMI of the participants were $19( \pm 3.1)$ and $19.8( \pm 3.1)$ respectively. The base line median values for CD4 count and Hgb were $280(\mathrm{IQR}=150-410)$ and 11 $(\mathrm{IQR}=9.8-13)$ respectively and their end line median values were 384 $(\mathrm{IQR}=237-478)$ and $12.5(\mathrm{IQR}=11-13.5)$ respectively.

More than four-fifth of the study participants were taking prophylaxis both at base line $(443,82.6 \%)$ and at follow up $(453,84.5 \%)$ and almost all of them had good drug adherence status both at base line $(415,93.7 \%)$ and at follow up $(422,93.2 \%)$.

Almost all of the participants (423, 95.5\%) were taking Cotrimoxazole prophylaxis both at base line and at follow up (439, 96.7\%).

Half of the study participants $(268,50 \%)$ were taking ART both at base line and at follow up. All participants were on first line ART regimens in which about one third were taking TDF+3TC+EFV regimen both at base line $(101,37.7 \%)$ and at follow up $(106,39.6 \%)$. Almost all study participants had good antiretroviral drug adherence both at base line $(252,94 \%)$ and follow up $(253,94.4 \%)$ (Table 3$)$.

\begin{tabular}{|c|c|c|c|c|c|}
\hline $\begin{array}{c}\text { Interval } \\
\text { start time }\end{array}$ & $\begin{array}{l}\text { Number } \\
\text { entering } \\
\text { interval }\end{array}$ & $\begin{array}{c}\text { Number } \\
\text { withdrawing } \\
\text { during interval }\end{array}$ & $\begin{array}{c}\text { Number } \\
\text { of any Ols } \\
\text { recurred }\end{array}$ & $\begin{array}{c}\text { Cumulative } \\
\text { proportion surviving } \\
\text { at end of interval }\end{array}$ & $\begin{array}{c}\text { Hazard } \\
\text { Rate }\end{array}$ \\
\hline 0 & 536 & 16 & 38 & .93 & .01 \\
\hline 10 & 482 & 12 & 63 & .81 & .01 \\
\hline 20 & 407 & 20 & 41 & .72 & .01 \\
\hline 30 & 346 & 17 & 35 & .65 & .01 \\
\hline 40 & 294 & 12 & 38 & .56 & .01 \\
\hline 50 & 244 & 16 & 32 & .49 & .01 \\
\hline 60 & 196 & 8 & 33 & .40 & .02 \\
\hline 70 & 155 & 4 & 29 & .33 & .02 \\
\hline 80 & 122 & 6 & 21 & .27 & .02 \\
\hline 90 & 95 & 2 & 6 & .25 & .01 \\
\hline 100 & 87 & 1 & 14 & .21 & .02 \\
\hline 110 & 72 & 2 & 7 & .19 & .01 \\
\hline 120 & 63 & 2 & 6 & .17 & .01 \\
\hline 130 & 55 & 0 & 5 & .16 & .01 \\
\hline 140 & 50 & 2 & 4 & .14 & .01 \\
\hline 150 & 44 & 1 & 6 & .12 & .01 \\
\hline 160 & 37 & 0 & 7 & .10 & .02 \\
\hline 170 & 30 & 3 & 6 & .08 & .02 \\
\hline 180 & 21 & 0 & 4 & .06 & .02 \\
\hline 190 & 17 & 0 & 0 & .06 & .00 \\
\hline 200 & 17 & 2 & 4 & .05 & .03 \\
\hline 210 & 11 & 2 & 1 & .04 & .01 \\
\hline 220 & 8 & 1 & 3 & .03 & .05 \\
\hline 230 & 4 & 0 & 1 & .02 & .03 \\
\hline 240 & 3 & 0 & 1 & .01 & .04 \\
\hline 250 & 2 & 1 & 1 & .00 & .10 \\
\hline
\end{tabular}

Table 2: The actuarial life table estimation of cumulative progression to recurrence of any OI in PLHIV in Debre Markos Hospital and Health Center, Northwest Ethiopia, between $25^{\text {th }}$ March 2007 and $24^{\text {th }}$ March, 2013. 
Citation: Mellie H, Mitike G, Abajobir AA (2013) Time to Recurrence of any Opportunistic Infection after Treatment of it among People Living with HIV Infection in Debre Markos, Northwest Ethiopia: Retrospective Cohort Study. J AIDS Clin Res 4: 226. doi:10.4172/2155-6113.1000226

Page 5 of 10

\begin{tabular}{|c|c|c|c|c|c|c|}
\hline \multirow[t]{2}{*}{ Characteristics } & \multicolumn{2}{|c|}{ Any OI recurred } & \multirow[b]{2}{*}{ Total (\%) } & \multirow{2}{*}{$\begin{array}{c}\text { Log rank test } \\
p \text {-value }\end{array}$} & \multirow{2}{*}{ CHR $[95 \% \mathrm{Cl}]$} & \multirow{2}{*}{$\begin{array}{l}p \text {-value } \\
\text { for CHR }\end{array}$} \\
\hline & Yes (\%) & No (\%) & & & & \\
\hline $\begin{array}{l}\text { Marital status } \\
\text { Married } \\
\text { Single } \\
\text { Divorced } \\
\text { Widowed }\end{array}$ & $\begin{array}{r}172(42.4) \\
85(20.9) \\
103(25.4) \\
46(11.3)\end{array}$ & $\begin{array}{l}60(46.2) \\
17(13.0) \\
37(28.5) \\
16(12.3)\end{array}$ & $\begin{array}{r}232(43.3) \\
102(19.0) \\
140(26.1) \\
62(11.6)\end{array}$ & 0.001 & $\begin{array}{c}1 \\
1.42(1.09-1.84) \\
1.11(0.87-1.41) \\
1.83(1.32-2.54)\end{array}$ & $\begin{array}{l}0.0088^{*} \\
0.4236 \\
0.0003^{*}\end{array}$ \\
\hline $\begin{array}{l}\text { Educational status } \\
\text { Not educated } \\
\text { Primary } \\
\text { Secondary } \\
\text { Tertiary }\end{array}$ & $\begin{array}{c}184(45.3) \\
122(30.1) \\
71(17.5) \\
29(7.1)\end{array}$ & $\begin{array}{l}52(40.0) \\
28(21.5) \\
24(18.5) \\
26(20.0)\end{array}$ & $\begin{array}{r}236(44.4) \\
150(27.6) \\
95(17.7) \\
55(10.3)\end{array}$ & $<0.0001$ & $\begin{array}{c}1 \\
0.92(0.73-1.16) \\
0.74(0.56-0.98) \\
0.45(0.31-0.68)\end{array}$ & $\begin{array}{c}0.461 \\
0.038^{*} \\
<0.0001^{*}\end{array}$ \\
\hline $\begin{array}{l}\text { Occupational status } \\
\text { Farmer } \\
\text { Merchant } \\
\text { Employed } \\
\text { Daily laborer } \\
\text { House wife } \\
\text { Unemployed } \\
\text { Student }\end{array}$ & $\begin{array}{l}73(18.0) \\
69(17.0) \\
69(17.0) \\
68(16.7) \\
42(10.3) \\
70(17.3) \\
15(3.7)\end{array}$ & $\begin{array}{c}25(19.2) \\
19(14.6) \\
38(29.2) \\
17(13.1) \\
11(8.5) \\
18(13.9) \\
2(1.5)\end{array}$ & $\begin{array}{r}98(18.3) \\
88(16.4) \\
107(20.0) \\
85(15.9) \\
53(9.9) \\
88(16.4) \\
17(3.2)\end{array}$ & $<0.0001$ & $\begin{array}{c}1 \\
0.43(0.31-0.60) \\
0.35(0.25-0.48) \\
0.86(0.62-1.21) \\
0.66(0.45-0.97) \\
0.92(0.66-1.28) \\
0.58(0.33-1.01)\end{array}$ & $\begin{array}{c}<0.0001^{*} \\
<0.0001^{*} \\
0.390 \\
0.034^{*} \\
0.612 \\
0.055\end{array}$ \\
\hline $\begin{array}{l}\text { CD4 count (cells/ul) } \\
<=100 \\
101-199 \\
200-350 \\
351-499 \\
>=500\end{array}$ & $\begin{array}{c}25(6.2) \\
34(8.4) \\
80(19.7) \\
124(30.5) \\
143(35.2)\end{array}$ & $\begin{array}{l}10(7.7) \\
14(10.8) \\
21(16.1) \\
35(26.9) \\
50(38.5)\end{array}$ & $\begin{array}{r}35(6.5) \\
48(9.0) \\
101(18.8) \\
159(29.7) \\
193(36.0)\end{array}$ & 0.04 & $\begin{array}{c}1 \\
0.45(0.27-0.77) \\
0.68(0.43-1.07) \\
0.73(0.48-1.13) \\
0.57(0.37-0.87)\end{array}$ & $\begin{array}{l}0.003^{*} \\
0.096 \\
0.158 \\
0.010^{*}\end{array}$ \\
\hline $\begin{array}{l}\text { Hemoglobin }(\mathrm{g} / \mathrm{dl})^{\mathrm{B} .} \\
<10 \\
>=10\end{array}$ & $\begin{array}{l}120(36.4) \\
210(63.6)\end{array}$ & $\begin{array}{l}14(21.9) \\
50(78.1)\end{array}$ & $\begin{array}{l}134(34.0) \\
260(66.0)\end{array}$ & $<0.0001$ & $\begin{array}{c}1 \\
0.56(0.45-0.70)\end{array}$ & $<0.0001^{*}$ \\
\hline $\begin{array}{l}\text { Functional status }{ }^{\mathrm{B} .} \\
\text { Working } \\
\text { Ambulatory } \\
\text { Bed ridden }\end{array}$ & $\begin{array}{c}307(75.6) \\
88(21.7) \\
11(2.7)\end{array}$ & $\begin{array}{c}111(85.4) \\
19(14.6) \\
-\end{array}$ & $\begin{array}{c}418(78.0) \\
107(20.0) \\
11(2.0)\end{array}$ & 0.044 & $\begin{array}{c}1 \\
1.32(1.09-1.67) \\
1.43(0.72-2.61)\end{array}$ & $\begin{array}{l}0.0226^{*} \\
0.2488\end{array}$ \\
\hline $\begin{array}{l}\text { Prophylaxis exposure }{ }^{\mathrm{B} .} \\
\text { No } \\
\text { Yes }\end{array}$ & $\begin{array}{r}72(17.7) \\
334(82.3)\end{array}$ & $\begin{array}{r}21(16.2) \\
109(83.8)\end{array}$ & $\begin{array}{r}93(17.4) \\
443(82.6)\end{array}$ & $<0.0001$ & $\begin{array}{c}1 \\
0.54(0.42-0.70)\end{array}$ & $<0.0001^{*}$ \\
\hline $\begin{array}{l}\text { Prophylaxis Adherence } \\
\text { Good } \\
\text { Fair } \\
\text { Poor }\end{array}$ & $\begin{array}{c}308(92.2) \\
11(3.3) \\
15(4.5)\end{array}$ & $\begin{array}{c}107(98.2) \\
- \\
2(1.8)\end{array}$ & $\begin{array}{c}415(93.7) \\
11(2.5) \\
17(3.8)\end{array}$ & 0.001 & $\begin{array}{c}1 \\
1.56(0.85-2.44) \\
2.42(1.43-4.09)\end{array}$ & $\begin{array}{l}0.1506 \\
0.001^{*}\end{array}$ \\
\hline $\begin{array}{l}\text { ART }{ }^{\mathrm{B}} \\
\text { No } \\
\text { Yes }\end{array}$ & $\begin{array}{l}200(49.3) \\
206(50.7)\end{array}$ & $\begin{array}{l}68(52.3) \\
62(47.7)\end{array}$ & $\begin{array}{l}268(50.0) \\
268(50.0)\end{array}$ & $<0.0001$ & $\begin{array}{c}1 \\
0.68(0.56-0.83)\end{array}$ & $<0.0001^{*}$ \\
\hline $\begin{array}{c}\text { ART regimens } ¥ \mathrm{~B} . \\
\mathrm{D} 4 \mathrm{~T}+3 \mathrm{TC}+\mathrm{NVP} \\
\mathrm{D} 4 \mathrm{~T}+3 \mathrm{TC}+\mathrm{EFV} \\
\mathrm{AZT}+3 \mathrm{TC}+\mathrm{NVP} \\
\text { AZT+3TC+EFV } \\
\text { TDF+3TC+EFV } \\
\mathrm{TDF}+3 T C+N V P\end{array}$ & $\begin{array}{c}55(26.7) \\
19(9.3) \\
60(29.1) \\
11(5.3) \\
61(29.6) \\
-\end{array}$ & $\begin{array}{c}7(11.3) \\
2(3.2) \\
6(9.7) \\
1(1.6) \\
41(66.1) \\
5(8.1)\end{array}$ & $\begin{array}{c}62(23.1) \\
21(7.8) \\
66(24.6) \\
12(4.6) \\
101(37.7) \\
6(2.2)\end{array}$ & 0.088 & $\begin{array}{c}1 \\
1.00(0.59-1.69) \\
1.20(0.83-1.74) \\
1.89(0.98-3.64) \\
1.58(1.08-2.32) \\
0.01(0.001-1.10)\end{array}$ & $\begin{array}{c}0.9999 \\
0.3311 \\
0.0566 \\
0.0197^{*} \\
0.9881\end{array}$ \\
\hline $\begin{array}{l}\text { ART adherence }{ }^{\text {B. }} \\
\text { Good } \\
\text { Fair } \\
\text { Poor }\end{array}$ & $\begin{array}{c}194(94.2) \\
5(2.4) \\
7(3.4)\end{array}$ & $\begin{array}{c}61(98.4) \\
1(1.6) \\
-\end{array}$ & $\begin{array}{c}255(95.2) \\
6(2.2) \\
7(2.6)\end{array}$ & $<0.0001$ & $\begin{array}{c}1 \\
2.15(0.88-5.25) \\
3.89(1.81-8.36)\end{array}$ & $\begin{array}{c}0.092 \\
<0.0001^{*}\end{array}$ \\
\hline $\begin{array}{l}\text { Body mass index }\left(\mathrm{kg} / \mathrm{m}^{2}\right)^{\mathrm{F}} \\
<=18.4 \\
18.5-22.9 \\
>=23\end{array}$ & $\begin{array}{r}151(37.2) \\
196(48.3) \\
59(14.5)\end{array}$ & $\begin{array}{l}38(29.2) \\
64(49.3) \\
28(21.5)\end{array}$ & $\begin{array}{r}189(35.3) \\
260(48.5) \\
87(16.2)\end{array}$ & 0.008 & $\begin{array}{c}1 \\
0.87(0.71-1.08) \\
0.62(0.46-0.84)\end{array}$ & $\begin{array}{l}0.2142 \\
0.0022^{*}\end{array}$ \\
\hline  & $\begin{array}{l}13(4.8) \\
31(11.4) \\
93(34.3) \\
75(27.7) \\
59(21.8)\end{array}$ & $\begin{array}{c}5(5.7) \\
7(8.0) \\
33(37.9) \\
26(29.9) \\
16(18.5)\end{array}$ & $\begin{array}{r}18(5.0) \\
38(10.7) \\
126(35.2) \\
101(28.2) \\
75(20.9)\end{array}$ & 0.55 & $\begin{array}{c}1 \\
0.58(0.30-1.12) \\
0.53(0.29-0.94) \\
0.46(0.25-0.83) \\
0.43(0.23-0.79)\end{array}$ & $\begin{array}{l}0.104 \\
0.031^{*} \\
0.010^{*} \\
0.006^{*}\end{array}$ \\
\hline $\begin{array}{l}\text { Functional status }{ }^{\mathrm{F}} \\
\text { Working } \\
\text { Ambulatory } \\
\text { Bed ridden }\end{array}$ & $\begin{array}{c}345(85.0) \\
51(12.5) \\
10(2.5)\end{array}$ & $\begin{array}{c}126(96.9) \\
3(2.3) \\
1(0.8)\end{array}$ & $\begin{array}{c}471(87.9) \\
54(10.1) \\
11(2.0)\end{array}$ & 0.006 & $\begin{array}{c}1 \\
1.59(1.18-2.13) \\
1.32(0.70-2.47)\end{array}$ & $\begin{array}{l}0.0021^{*} \\
0.3909\end{array}$ \\
\hline $\begin{array}{l}\text { Prophylaxis exposure }{ }^{F} \\
\text { No } \\
\text { Yes }\end{array}$ & $\begin{array}{r}63(15.5) \\
343(84.5)\end{array}$ & $\begin{array}{r}20(15.4) \\
110(84.6)\end{array}$ & $\begin{array}{r}83(15.5) \\
453(84.5)\end{array}$ & 0.003 & $\begin{array}{c}1 \\
0.67(0.51-0.87)\end{array}$ & $0.0032^{*}$ \\
\hline
\end{tabular}


Citation: Mellie H, Mitike G, Abajobir AA (2013) Time to Recurrence of any Opportunistic Infection after Treatment of it among People Living with HIV Infection in Debre Markos, Northwest Ethiopia: Retrospective Cohort Study. J AIDS Clin Res 4: 226. doi:10.4172/2155-6113.1000226

Page 6 of 10

\begin{tabular}{|c|c|c|c|c|c|c|}
\hline $\begin{array}{l}\text { Prophylaxis Adherence } \mathrm{F} . \\
\text { Good } \\
\text { Fair } \\
\text { Poor }\end{array}$ & $\begin{array}{c}316(92.1) \\
10(2.9) \\
17(5.0)\end{array}$ & $\begin{array}{r}108(98.2) \\
1(0.9) \\
1(0.9)\end{array}$ & $\begin{array}{c}424(93.6) \\
11(2.4) \\
18(4)\end{array}$ & $<0.0001$ & $\begin{array}{c}1 \\
1.49(0.79-2.79) \\
3.48(2.12-5.71)\end{array}$ & $\begin{array}{c}0.217 \\
<0.0001^{*}\end{array}$ \\
\hline $\begin{array}{l}\text { ART F. } \\
\text { No } \\
\text { Yes }\end{array}$ & $\begin{array}{l}200(49.3) \\
206(50.7)\end{array}$ & $\begin{array}{l}68(52.3) \\
62(47.7)\end{array}$ & $\begin{array}{l}268(50) \\
268(50)\end{array}$ & $<0.0001$ & $\begin{array}{c}1 \\
0.68(0.56-0.83)\end{array}$ & $<0.0001^{*}$ \\
\hline
\end{tabular}

B.= at base line

$\mathrm{F}=$ at Follow up

*significant at $\alpha=5 \%$

CHR=crude hazard rate

$¥$ d4T: Stavudine; 3TC: Lamivudine; NVP: Nevirapine; EFV: Efavirenz; AZT:Zidouvudine; TDF: Tenofovir disoproxil fumarate

Table 3: Bivariate cox proportional hazard model of the association between base line and follow up characteristics and any OI recurrence in among PLHIV in Debre Markos Hospital and Health Center, Northwest Ethiopia, between 25 March 2007 and 24 March, 2013.



Figure 2: kaplan-meier survival estimation of time to free of any OI recurrence in PLHIV in Debre Markos Hospital and Health Center, Northwest Ethiopia, between $25^{\text {th }}$ March 2007 and 24 ${ }^{\text {th }}$ March, 2013.

\section{Magnitude of any OI recurrence}

During follow up, OIs recurred in three quarter study participants $406(75.7 \%)$ and the rest were censored $130(24.3 \%)$. Proportion of recurrence was 200 (74.6\%) in Pre-ART and 206 (76.9\%) ART PLHIV.

In chi-square test, the proportion of recurrence was not statistically significant different in the overall or each type of OI in Pre-ART and ART PLHIV.

The overall incidence rate of any OI recurrence was 1.31 per 100 person weeks and it was 1.57 and 1.13 per 100 person weeks in pre-ART and ART PLHIV respectively. In the study area, frequently recurred OIs were recurrent upper respiratory tract infection 73 (13.6\%), chronic diarrhea $52(9.7 \%)$, pneumonia $46(8.5 \%)$, oral candidiasis $43(8 \%)$, herpes zoster 38 (7.1\%) though their proportions and the commonly recurred OIs were different in pre-ART and ART PLHIV (Table 1).

\section{Survival analysis}

A total of 536 PLHIV were followed for a median of 43 (IQR=20.2575.75) person weeks. The minimum, maximum and total person weeks follow up was 2, 258 and 30971 respectively. According to the Kaplanmeier survival estimation, the median time of survival was 57 weeks (95\%CI: 51.66-62.34). The actuarial life table analysis showed that most $(63,11.8 \%)$ OIs recurred from $10-20$ weeks. The cumulative proportion of free from any OI recurrence up to week 20 was $81 \%$. The cumulative proportion of free from any OI recurrence up to week 250 was $1 \%$ (Table 2). 
The median time of survival was different in pre-ART and ART PLHIV, that was 52 (95\%CI: 45.24-58.76) and 64 (95\% CI: 54.87-73.13) weeks respectively (Figures 2 and 3).

At base line and follow up, significant difference in time to recurrence of any $\mathrm{OI}$ in log rank test among categorical variables strata were seen in marital status $(\mathrm{p}=0.001)$, educational status $(\mathrm{p}<0.0001)$, occupational status $(p<0.0001)$, base line CD4 count $(p=0.0084)$, base line Hgb value $(\mathrm{p}<0.0001)$, follow up Hgb value $(\mathrm{p}=0.01)$, follow up BMI ( $\mathrm{p}=0.008)$, base line functional status $(\mathrm{P}=0.044)$, follow up functional status $(\mathrm{p}=0.006)$, ART exposure status $(\mathrm{p}<0.0001)$, base line prophylaxis exposure $(\mathrm{p}<0.0001)$, follow up prophylaxis exposure $(\mathrm{p}=0.003)$, base line ART adherence status $(\mathrm{p}<0.0001)$, follow up ART adherence status $(\mathrm{p}<0.0001)$, base line prophylaxis adherence status $(\mathrm{p}=0.001)$ and follow up prophylaxis adherence status $(\mathrm{p}<0.0001)$.

In univariate cox-regression analysis, the base line and follow up characteristics were significantly $(\mathrm{p}<0.05)$ associated with survival; namely marital status, educational status, occupational status, base line and follow up CD4 count, base line and follow up Hgb value, follow up BMI, base line and follow up functional status, ART exposure, base line and follow up prophylaxis exposure, base line and follow up ART adherence status, base line ART regimen, base line and follow up prophylaxis adherence status (Table 3).

In Multivariable cox proportional hazard model, only those variables which were associated with survival with $\mathrm{p}$-value $\leq 0.2$ and not collinear were entered to the final model. After adjustment for covariates, significant predictors reducing survival (risks for recurrence) were being widowed than married by 2.18 times, fair follow up prophylaxis adherence than good adherence by 7.27 times, fair base line ART adherence than good adherence by 14.83 times. The significant predictors that improve survival (prevent recurrence) were divorced than married by 0.57 times, compared to farmer being merchant, employed and student by $0.30,0.22$ and 0.15 times respectively, having base line $\mathrm{Hgb}$ value of $\geq \mathrm{g} / \mathrm{dl}$ than $<10$ by 0.42 times, compared $\leq 100$ cells/ $\mu \mathrm{l}$ CD4 count having 351-499 and $\geq 500$ follow up CD4 count by 0.39 and 0.32 times respectively; moreover, taking prophylaxis at baseline, at follow up and taking ART by 0.55 and 0.49 by 0.522 times respectively. After adjusting for other variables, when follow up CD4 count increased by 50 cells/ $\mu$ survival also increased by 0.91 times $(\mathrm{p}<0.0001)$, and when base line Hgb value increased by $5 \mathrm{~g} / \mathrm{dl}$ survival also increased by 0.66 times $(\mathrm{p}=0.004)$ (Table 4$)$.

\section{Discussion}

In this historical cohort study, more than three quarter of the participants had recurrence of OIs. Proportion of recurrence was not significantly different $(\mathrm{p}=0.614)$ between Pre-ART and ART PLHIV. The proportion of OI recurrence in pre-ART PLHIV was slightly lower than the study in Northwest Ethiopia in which $82.4 \%$ patients present with any type of OIs at start of ART [15] and in other study in Felegehiot hospital among pre-ART HIV patients, it was $88.9 \%$ at

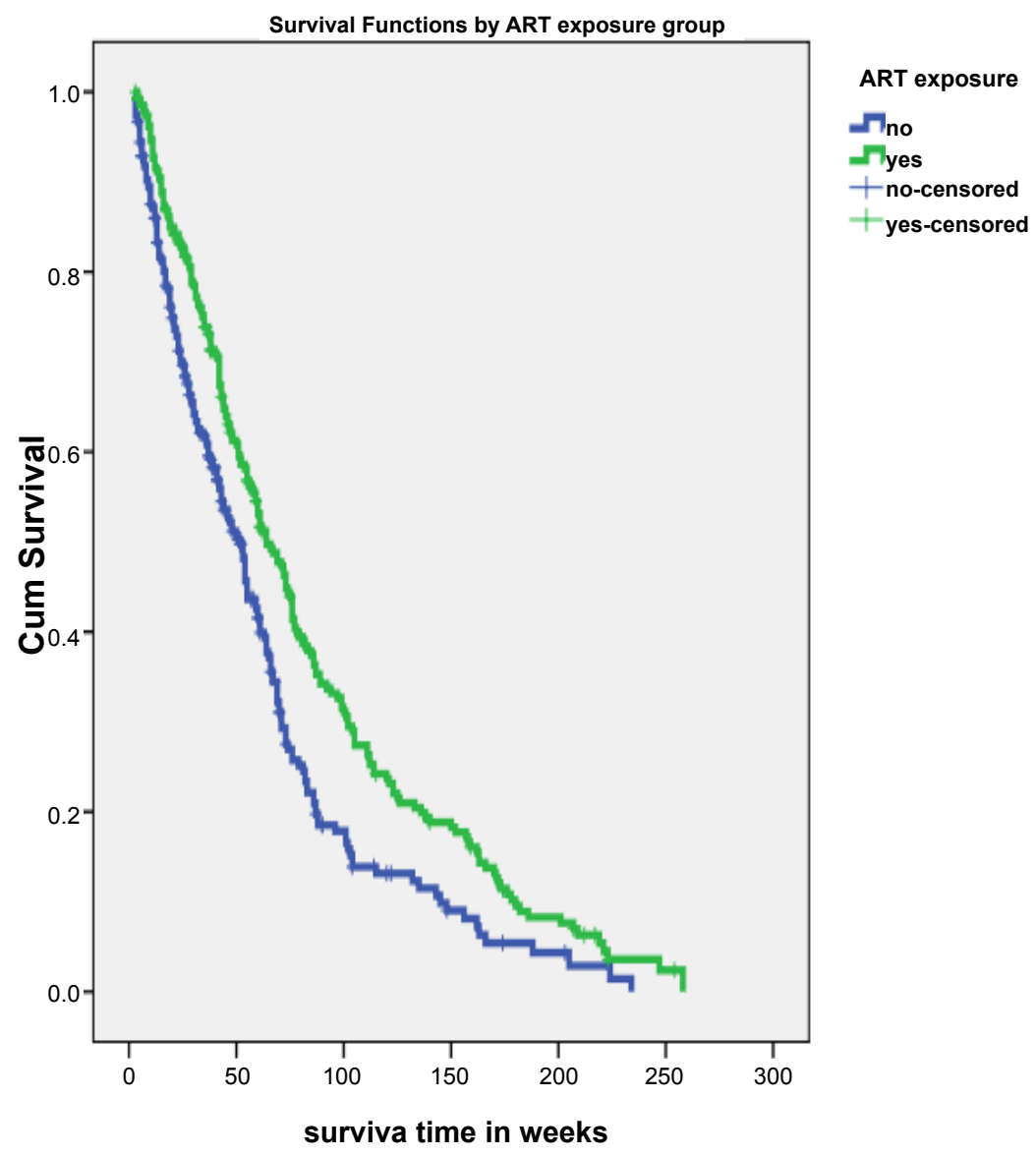

Figure 3: kaplan-meier survival estimation of time to free of any OI recurrence in pre-ART and ART PLHIV in Debre Markos Hospital and Health Center, Northwest Ethiopia, between $25^{\text {th }}$ March 2007 and $24^{\text {th }}$ March, 
Citation: Mellie H, Mitike G, Abajobir AA (2013) Time to Recurrence of any Opportunistic Infection after Treatment of it among People Living with HIV Infection in Debre Markos, Northwest Ethiopia: Retrospective Cohort Study. J AIDS Clin Res 4: 226. doi:10.4172/2155-6113.1000226

Page 8 of 10

\begin{tabular}{|c|c|c|c|c|}
\hline Characteristics & AHR $[95 \% \mathrm{Cl}]$ & \multicolumn{3}{|l|}{ p-value } \\
\hline $\begin{array}{l}\text { Marital status } \\
\text { Married } \\
\text { Single } \\
\text { Divorced } \\
\text { Widowed }\end{array}$ & $\begin{array}{l}1 \\
1.25(0.83-1.87) \\
0.57(0.38-0.84) \\
2.18(1.30-3.64)\end{array}$ & $\begin{array}{l}0.287 \\
0.005^{*} \\
0.003^{*}\end{array}$ & & \\
\hline $\begin{array}{l}\text { Educational status } \\
\text { Not educated } \\
\text { Primary } \\
\text { Secondary } \\
\text { Tertiary }\end{array}$ & $\begin{array}{l}1 \\
1.34(0.90-1.99) \\
1.09(0.66-1.79) \\
1.05(0.49-2.21)\end{array}$ & $\begin{array}{l}0.151 \\
0.747 \\
0.898\end{array}$ & & \\
\hline $\begin{array}{l}\text { Occupational status } \\
\text { Farmer } \\
\text { Merchant } \\
\text { Employed } \\
\text { Daily laborer } \\
\text { House wife } \\
\text { Unemployed } \\
\text { Student }\end{array}$ & $\begin{array}{l}1 \\
0.30(0.17-0.53) \\
0.22(0.12-0.40) \\
0.79(0.47-1.35) \\
0.55(0.29-1.03) \\
0.59(0.35-1.03) \\
0.15(0.06-0.38)\end{array}$ & $\begin{array}{l}<0.0001^{*} \\
<0.0001^{*} \\
0.388 \\
0.058 \\
0.063 \\
<0.000^{\star}\end{array}$ & & \\
\hline $\begin{array}{l}\text { Follow up body mass index }\left(\mathrm{kg} / \mathrm{m}^{2}\right) \\
<=18.4 \\
18.5-22.9 \\
>=23\end{array}$ & $\begin{array}{l}1 \\
1.05(0.74-1.48) \\
0.82(0.51-1.31)\end{array}$ & $\begin{array}{l}0.805 \\
0.400\end{array}$ & & \\
\hline $\begin{array}{l}\text { ART } \\
\text { No } \\
\text { Yes }\end{array}$ & $\begin{array}{l}1 \\
0.52(0.35-0.78)\end{array}$ & $<0.002^{*}$ & & \\
\hline $\begin{array}{l}\text { Follow up ART regimens } ¥ \\
\text { D4T+3TC+NVP } \\
\text { D4T+3TC+EFV } \\
\text { AZT+3TC+NVP } \\
\text { AZT+3TC+EFV } \\
\text { TDF+3TC+EFV } \\
\text { TDF+3TC+NVP }\end{array}$ & $\begin{array}{l}1 \\
0.45(0.15-1.36) \\
0.64(0.32-1.29) \\
1.14(0.27-4.82) \\
0.47(0.22-1.01) \\
1.33(0.21-8.55)\end{array}$ & $\begin{array}{l}0.157 \\
0.211 \\
0.861 \\
0.053 \\
0.763\end{array}$ & & \\
\hline & Base line & & Follow up & \\
\hline & AHR $[95 \% \mathrm{Cl}]$ & $p$-value & AHR $[95 \% \mathrm{Cl}]$ & $p$-value \\
\hline $\begin{array}{l}\text { CD4 count }(\text { cells } / \mu \mathrm{l}) \\
<=100 \\
101-199 \\
200-350 \\
351-499 \\
>=500\end{array}$ & $\begin{array}{l}1 \\
0.46(0.19-1.13) \\
0.59(0.26-1.31) \\
0.60(0.26-1.43) \\
0.52(0.22-0.25)\end{array}$ & $\begin{array}{l}0.089 \\
0.191 \\
0.251 \\
0.142\end{array}$ & $\begin{array}{l}1 \\
0.56(0.24-1.31) \\
0.68(0.31-1.49) \\
0.39(0.17-0.87) \\
0.32(0.14-0.73)\end{array}$ & $\begin{array}{l}0.182 \\
0.338 \\
0.022^{*} \\
0.007^{\star}\end{array}$ \\
\hline $\begin{array}{l}\text { Functional status } \\
\text { Working } \\
\text { Ambulatory } \\
\text { Bed ridden }\end{array}$ & $\begin{array}{l}1 \\
1.99(0.67-1.47) \\
0.98(0.36-2.65)\end{array}$ & $\begin{array}{l}0.961 \\
0.965\end{array}$ & $\begin{array}{l}1 \\
1.12(0.67-1.86) \\
1.49(0.57-3.93)\end{array}$ & $\begin{array}{l}0.664 \\
0.414\end{array}$ \\
\hline $\begin{array}{l}\text { Prophylaxis exposure } \\
\text { No } \\
\text { Yes }\end{array}$ & $\begin{array}{l}1 \\
0.55(0.33-0.91)\end{array}$ & $0.021^{*}$ & $0.49(0.28-0.87)$ & $0.014^{*}$ \\
\hline $\begin{array}{l}\text { Prophylaxis Adherence } \\
\text { Good } \\
\text { Fair } \\
\text { Poor }\end{array}$ & $\begin{array}{l}1 \\
0.92(0.16-5.41) \\
2.96(0.31-28.14)\end{array}$ & $\begin{array}{l}0.925 \\
0.346\end{array}$ & $\begin{array}{l}1 \\
7.27(1.31-40.45) \\
3.59(0.52-22.69)\end{array}$ & $\begin{array}{l}0.023^{*} \\
0.194\end{array}$ \\
\hline $\begin{array}{l}\text { ART adherence } \\
\text { Good } \\
\text { Fair } \\
\text { Poor }\end{array}$ & $\begin{array}{l}1 \\
14.83(1.86-12.41) \\
6.64(0.31-144.60)\end{array}$ & $\begin{array}{l}0.011^{*} \\
0.229\end{array}$ & $\begin{array}{l}1 \\
1.91(0.38-9.67) \\
1.81(0.09-36.34)\end{array}$ & $\begin{array}{l}0.435 \\
0.697\end{array}$ \\
\hline
\end{tabular}

*significant at $\alpha=5 \%$

AHR=adjusted hazard rate

$¥ \mathrm{~d} 4 \mathrm{~T}$ : Stavudine; 3TC: Lamivudine; NVP: Nevirapine; EFV: Efavirenz; AZT:

Zidouvudine; TDF: Tenofovir disoproxil fumarate

Table 4: Multivariate cox proportional hazard model of the association between base line and follow up characteristics and any OI recurrence in study participants' in Debre Markos Hospital and Health Center, Northwest Ethiopia, between 25 March 2007 and 24 March, 2013.

ART enrolment [9]. The smaller in magnitude was due to inclusion of all Pre-ART PLHIV who were on chronic HIV care and treated for any OIs but the previous studies [9,15] assessed only Pre-ART PLHIV who were enrolled for ART which might increase the magnitude because ART enrollment was done using WHO clinical staging and CD4 count.

This study showed taking ART reduces time to any OI recurrence which is supported by different studies [12,13,16-19]. The one year follow-up study after ART initiation in Northwest Ethiopia showed ART reduces any OIs occurrence by $91.9 \%$. HAART reduces the incidence of severe OIs by $21 \%$ during the first 6 months and $28 \%$ during subsequent follow up after adjusting for CD4 count [19].

Antiretroviral treatment adherence is one of the challenges in the 
Citation: Mellie H, Mitike G, Abajobir AA (2013) Time to Recurrence of any Opportunistic Infection after Treatment of it among People Living with HIV Infection in Debre Markos, Northwest Ethiopia: Retrospective Cohort Study. J AIDS Clin Res 4: 226. doi:10.4172/2155-6113.1000226

Page 9 of 10

failure of HAART increasing the chance for OI progression $[9,20]$. This study revealed that base line fair ART adherence compared to good adherence was hazard for survival illustrating adherence is crucial to improving survival besides taking ART.

This study revealed that survival was enhanced by taking prophylaxis at baseline and at follow up similar with other studies [21-24]. A critical appraisal in 2011 showed primary prophylaxis with Trimethoprim-sulfamethoxazole is preventing life-threatening OIs like PCP, toxoplasmosis and bacterial infections [21]. Primary prophylaxis with anti-tuberculosis regimens seems more effective at reducing the incidence of active tuberculosis in PLHIV [22]. An experimental study in Ugandan adults with Cotrimoxazole prophylaxis showed its effect on prevention of OIs like diarrhea in PLHIV. Compared to those remaining on cotrimoxazole, patients who discontinued had a relative risk of diarrhea of 1.8 and at least 1 episode of diarrhea occurred in $14 \%$ among those continued versus $25 \%$ in those discontinued. Participants who discontinued cotrimoxazole were more likely to be diagnosed with a respiratory tract infection [23]. Another study also showed Cotrimoxazole is preventing PCP $(\mathrm{RR}=0.59, \mathrm{p}=0.03)$ [24].

This study revealed having higher follow up CD4 count prolongs survival, compared $\leq 100$ cells/ $\mu \mathrm{l}$; this result is in conformity with previous studies $[13,18,19,25,26]$. Similar cohort study also showed the risk of toxoplasmic encephalitis occurrence was increased by $30 \%$ for each 50-cell decrease in CD4+ cell count, independent of antiretroviral [13].

\section{Conclusions}

Since the introduction of free ART, more patients have been presented to HIV care and support. A number of PLHIV seeking HIV care are increasing through time in health institutions of Debre Markos to get the services. During a median of 43 person weeks follow up OIs were recurred in three quarter study participants (75.7\%). The overall incidence rate of any OI recurrence was 1.31 per 100 person weeks. Independent significant predictors that reduce recurrence (risk factors) and improve (preventive factors) survival were identified. Those risk factors were being widowed than married, non- adherence of ART and prophylaxis treatments; however, preventive factors were being divorced compared to married, having occupational status of merchant, employed and student compared to farmer, having $\geq 10 \mathrm{~g} / \mathrm{dl}$ Hgb value, having CD4 count 351 cells $/ \mu \mathrm{l}$ and above compared to $\leq 100$ cells $/ \mu \mathrm{l}$, taking ART and prophylaxis treatments.

\section{Recommendations}

\section{To hospitals and health centers with ART clinic (giving HIV care and support)}

$>$ PLHIV should be encouraged to take ART and prophylaxis drugs and counseled to adhere on them properly

$>$ Increasing the CD4 count and Hgb value, using nutrition and drug treatments are crucial to improve survival

$>$ Preventive efforts should focus on high risk groups such as widowed, not adhering ART or prophylaxis

$>$ The ART care should be further enhanced to improve survival.

To Debre Markos town health office and other responsible organizations

$>$ In-service training should be given for the healthcare provider on HIV/AIDS care and support especially on how to distinguish and manage patients with high risk

> Health professionals should be encouraged to properly document patients' healthcare data

$>$ Priority should be given for increasing job opportunities for PLHIV in governmental or non-governmental organizations or facilitate conditions to join other options.

\section{To research and academic institutions}

$>$ Further studies for estimating economic losses for subsequent recurrence of $\mathrm{OI}$ is recommended

$>$ Further observational studies with prospective design to ascertain the findings are recommended.

\section{Acknowledgments}

I would like to thank the School of Public Health of Addis Ababa for its financial support. Our sincere thanks also go to Debre Markos Referral hospital and health center staffs and the study participants

\section{References}

1. U.S. Global Health Policy Fact Sheet (2011) the Global HIVIAIDS Epidemic.

2. WHO (2011) Global HIVIAIDS Response: Epidemic update and health sector progress towards Universal Access WHO, UNICEF, UNAIDS.

3. UNAIDS (2012) Together we will end AIDS. Joint United Nations Programme on HIVIAIDS, Geneva.

4. Central Statistical Agency (CSA) (2011) Ethiopia Demographic and Health Survey; final draft report. Addis Ababa Ethiopia, ICF International Calverton, Maryland, USA.

5. Federal HIVIAIDS Prevention and Control Office (2010) Annual Performance Report of Multi-sectoral HIV/ AIDS Response, Federal HIVIAIDS Prevention and Control Office 2002 E.C.

6. Report on progress towards implementation of the UN Declaration of Commitment on HIVIAIDS. Federal Democratic Republic of Ethiopia Federal HIVIAIDS Prevention and Control Office.

7. Mariam ZT, Abebe G, Mulu A (2008) Opportunistic and other intestina parasitic infections in AIDS patients, HIV seropositive healthy carriers and HIV seronegative individuals in southwest Ethiopia. East Afr J Public Health 5: 169 173

8. Gallant JE, Moore RD, Chaisson RE (1994) Prophylaxis for opportunistic infections in patients with HIV infection. Ann Intern Med 120: 932-944.

9. Bayeh A, Tsehaye Tewabe, Atnaf Alem, Mohammed Yessin (2010) ART-naive HIV patients at Feleg-Hiwot Referral Hospital Northwest Ethiopia. Ethiop J Health Dev 24: 3-8.

10. Saha K, Firdaus R, Santra P, Pal J, Roy A, et al. (2011) Recent pattern of Coinfection amongst HIV seropositive individuals in tertiary care hospital, Kolkata. Virol J 8: 116 .

11. Manosuthi W, Chaovavanich A, Tansuphaswadikul S, Prasithsirikul W, Inthong $Y$, et al. (2007) Incidence and risk factors of major opportunistic infections after initiation of antiretroviral therapy among advanced HIV-infected patients in a resource-limited setting. J Infect 55: 464-469.

12. Detels R, Tarwater P, Phair JP, Margolick J, Riddler SA, et al. (2001) Effectiveness of potent antiretroviral therapies on the incidence of opportunistic infections before and after AIDS diagnosis. AIDS 15: 347-355.

13. Antinori A, Larussa D, Cingolani A, Lorenzini P, Bossolasco S, et al. (2004) Prevalence, Associated Factors, and Prognostic Determinants of AIDS-Related Toxoplasmic Encephalitis in the Era of Advanced Highly Active Antiretroviral Therapy, Italy. Clin Infect Dis 39:1681-1691.

14. Casagrande, Fleiss, Dupont (1990) sample size calculation for different study designs.

15. Nasir TW, Mohammed A (2012) Immunological and clinical progress of HIVinfected patients on highly active antiretroviral therapy in North West Ethiopia Gaziantep Medical Journal 18: 31-36.

16. Saka B, Landoh DE, Kombaté K, Mouhari-Toure A, Makawa MS, et al. (2012) 
Citation: Mellie H, Mitike G, Abajobir AA (2013) Time to Recurrence of any Opportunistic Infection after Treatment of it among People Living with HIV Infection in Debre Markos, Northwest Ethiopia: Retrospective Cohort Study. J AIDS Clin Res 4: 226. doi:10.4172/2155-6113.1000226

[Evaluation of antiretroviral treatment in a cohort of 1,620 HIV-infected patients in Togo]. Med Sante Trop 22: 193-197.

17. Modi B, Patel P, Patel S (2011) Aprofile of patients registered at art centre at surat municipal institute of medical education \& research in Surat city, Gujarat, India. LUNG DIS HIVIAIDS 8: 11-16.

18. Ledergerber B, Egger M, Erard V, Weber R, Hirschel B, et al. (1999) AIDSrelated opportunistic illnesses occurring after initiation of potent antiretrovira therapy: the Swiss HIV Cohort Study. JAMA 282: 2220-2226.

19. Losina E, Yazdanpanah Y, Deuffic-Burban S, Wang B, Wolf LL, et al. (2007) The Independent Effect of Highly Active Antiretroviral Therapy on Severe Opportunistic Disease Incidence and Mortality in HIV infected Adults in Côte d'Ivoire France. Antivir Ther 12: 543-551.

20. Sow PG, Toure K, Coume M, Dia AT, Traore I (2012) Predictors of ART adherence among HIV infected individuals in Dakar, Senegal. Journal of Medicine and Medical Science 3: 212-216.

21. Seddon J, Bhagani S (2011) Antimicrobial therapy for the treatment of opportunistic infections in HIVIAIDS patients: a critical appraisal. HIV AIDS (Auckl) 3: 19-33
22. Aberg J, Powderly W (2010) HIV: primary and secondary prophylaxis for opportunistic infections. Clin Evid (Online) 2010.

23. Campbell JD, Moore D, Degerman R, Kaharuza F, Were W, et al. (2012) HIVInfected Ugandan Adults Taking Antiretroviral Therapy With CD4 Counts $>200$ Cells/IL Who Discontinue Cotrimoxazole Prophylaxis Have Increased Risk of Malaria and Diarrhea. Clinical Infectious Diseases 54:1204-1211.

24. El-Sadr WM, Luskin-Hawk R, Yurik TM, Walker J, Abrams D, et al. (1999) A Randomized Trial of Daily and Thrice-Weekly Trimethoprim-Sulfamethoxazole for the Prevention of Pneumocystis carinii Pneumonia in Human Immunodeficiency Virus-Infected Persons. Clinica Infectious Disease 29: 775783

25. Egger M, May M, Chêne G, Phillips AN, Ledergerber B, et al. (2002) Prognosis of HIV-1-infected patients starting highly active antiretroviral therapy: a collaborative analysis of prospective studies. Lancet 360: 119-129.

26. Opportunistic Infections Project Team of the Collaboration of Observational HIV Epidemiological Research in Europe (COHERE) in EuroCoord, Young J Psichogiou M, Meyer L, Ayayi S, et al. (2012) CD4 cell count and the risk of AIDS or death in HIV-Infected adults on combination antiretroviral therapy with a suppressed viral load: a longitudinal cohort study from COHERE. PLoS Med 9: e1001194 